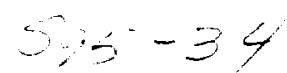

$\lambda$

\title{
WETTING HYSTERESIS AT THE MOLECULAR SCALE
}

\author{
Wei Jin ${ }^{1}$, Joel Koplik ${ }^{1}$ and Jayanth R. Banavar ${ }^{2}$ \\ ${ }^{1}$ Benjamin Levich Institute and Department of Physics, City College of the City University of \\ New York, New York, NY 10031 \\ ${ }^{2}$ Department of Physics and Materials Research Laboratory, Pennsylvania State University, \\ University Park, PA 16802
}

\begin{abstract}
The motion of a fluid-fluid-solid contact line on a rough surface is well known to display hysteresis in the contact angle vs. velocity relationship. In order to understand the phenomenon at a fundamental microscopic level, we have conducted molecular dynamics computer simulations of a Wilhelmy plate experiment in which a solid surface is dipped into a liquid bath, and the force-velocity characteristics are measured. We directly observe a systematic variation of force and contact angle with velocity, which is single-valued for the case of an atomically smooth solid surface. In the microscopically rough case, however, we find (as intuitively expected) an open hysteresis loop. Further characterization of the interface dynamics is in progress.
\end{abstract}

\section{INTRODUCTION}

The contact angle at which a meniscus separating two fluids meets a solid surface is an important quantity for both static and moving fluid interfaces. The static angle determines the shape of partially wetting drops and, when zero, implies that a drop will wet a surface completely. The dynamic contact angle of a moving interface is an important ingredient in its time evolution, and enters as a boundary condition in the free surface problem. In either case, a number of interesting issues arise, which are generically referred to as contact angle hysteresis $[1,2]$.

Firstly, Young's equation relates the contact angle to the various interfacial free energies in the problem, and ostensibly implies a unique angle for a given solid-liquid-liquid system. In fact, one observes that a range of static angles is possible. The origin of this phenomenon is believed to be surface heterogeneity, which can be of two varieties. If the surface is structurally rough, having a non-planar shape at mesoscopic lengths between the atomic size and the resolution of the observations, then even a unique microscopic or intrinsic angle on a fluctuating surface may give a range of apparent macroscopic angles. If instead, the surface has chemical heterogeneities, then the microscopic angle may fluctuate from point to point and cause the apparent angle to vary with the location of the interface. In the dynamic case, a second phenomenon arises - the observed contact angle varies with the velocity of the meniscus. Here, in addition to surface effects, the viscous stress resulting from fluid motion will certainly affect the interfacial shape away from the surface, and perhaps cause further changes in the apparent angle.

Although contact angle hysteresis has been well documented in the laboratory, and is a common ingredient in recent hydrodynamic calculations, its origins involve the interplay of fluid dynamics and microscopic surface effects and the difficulties of the problem have discouraged computational or theoretical studies of its origins. In terms of quantitative calculations, several authors have considered the possible static interfacial configurations resulting from model surface heterogeneities. The latter have ranged from a 2-d sinusoidal variation in the position of a solid surface [3] to a more realistic periodic pattern of wettability variation on a solid plate [4]. In the latter case, Schwartz and Garoff consider the effects of dipping the plate into a fluid bath, and find multiple minima in the free energy, deducing that the meniscus motion consists of alternating stick and jump 
events. Analytic time-dependent calculations of the origin of hysteresis have considered only the effects of a single defect - a localized region of different wettability [5], while molecular dynamics (MD) simulations aimed at the moving contact line singularity question [6] observed a systematic variation of contact angle with interface velocity.

In this paper we report on MD simulations of a Wilhelmy plate experiment, in which hysteresis is the focus. The Wilhelmy configuration of a plate dipped into a bath at fixed velocity directly gives the force as a function of plate velocity. Hysteresis appears as an open loop in the force-velocity plane as the plate velocity varies from positive to negative values. In this procedure, is a direct measurement of angle is not needed, although the force is easily converted to an apparent angle, which can be approximately correlated with observation. The advantage is that angles are difficult to observe directly in the laboratory at short distances from the solid, and difficult to quantify in molecular simulations due to small-system fluctuations. We consider structural heterogeneity only, and solid surfaces which are either atomically smooth, periodically rough, or randomly rough. We find that roughness is necessary to obtain hysteresis.

\section{SIMULATION METHOD}

Molecular calculations of fluid flow have become common [7], and the details of the present simulation are quite similar to that used previously in molecular studies of wetting processes $[6,8,9]$. In an MD calculation, one computes the (classical) motion of the individual atoms comprising the fluid by giving initial positions and velocities, and integrating Newton's equations of motion with a specified intermolecular potential. Since we are interested in fluid behavior near a solid wall, whose atomic size may be comparable to that of the fluid atoms, it is appropriate to treat the wall as a collection of atoms as well. A further consideration is that we wish the atoms to be as simple as possible so that typical correlation lengths are small compared to the size of the whole system, and continuum behavior may be expected. A soft-sphere atomic fluid with Lennard-Jones potentials is then optimal, but unfortunately leads to a rather diffuse interface [7], whose contact angle is difficult to establish. The interface may be sharpened up while retaining a monatomic fluid by considering the relative displacement of two immiscible viscous fluids, as in $[6,8]$, although chain molecules in contact with near-vacuum [9] would have the same effect.

The interaction between atoms is a two-body potential of generalized Lennard-Jones form,

$$
V_{i j}(r)=4 \epsilon\left[\left(\frac{r}{\sigma}\right)^{-12}-c_{i j}\left(\frac{r}{\sigma}\right)^{-6}\right]
$$

where $\epsilon$ and $\sigma$ are energy and length scales, respectively, and if $m$ is the (common) atomic mass of the fluids, the appropriate time scale is $\tau=\sigma \sqrt{m / \epsilon}$. All quantities discussed below are nondimensionalized using $\{\sigma, \tau, \epsilon\}$. The indices $i, j$ label the atomic species, fluid-1, fluid-2, confining walls $(\mathrm{W})$, and plate $(\mathrm{P})$, and the coefficient matrix $\left(c_{i j}\right)$ is chosen as follows. Between any two atoms of the same species, we use the standard interaction strength, $c_{i i}=1$ for all $i$. Atoms of different immiscible fluids should have a weaker attraction, and we choose $c_{12}=0$. The precise value controls the interfacial width, but we have not explored other choices. The fluid-plate interaction controls the wetting properties, and we choose the lower fluid to preferentially wet the plate, $c_{1 \mathrm{P}}=0.75$ and $c_{2 \mathrm{P}}=0.5$. The interaction of the fluids and the plate with the top and bottom walls is of little consequence, and we take $c_{1 \mathrm{w}}=c_{2 \mathrm{~W}}=c_{\mathrm{PW}}=0$. The remaining coefficients are determined by symmetry (Newton's third law). The $r^{-12}$ term provides a strong short-distance repulsion to prevent overlap of the atoms, and and we simply use the standard (unit) coefficient.

A snapshot of a typical simulated system is shown in Fig. 1, and is meant to mimic a laboratory Wilhelmy plate experiment. The system is fully three-dimensional but shown in a projected view for clarity. The two fluids have 11760 atoms each and are placed in a rectangular region of space with periodic boundary conditions in both horizontal directions. The fluids are confined in the vertical direction by solid walls and a solid plate straddles the fluid-fluid interface. The plate is periodic in 
the vertical direction as well as the direction normal to the figure, so as to allow as steady state to be established when it moves. Initially, the fluid atoms are placed on lattice sites with density 0.8 and given a random initial velocity chosen from a Boltzmann distribution at temperature $T=0.8$. After some time the atomic positions become disordered due to mutual interactions, although one sees some layering near the walls. The wall atoms are localized for all times on an approximate crystal structure by tethering them to the sites of a regular lattice by linear springs. In this way, one has a realistic solid structure while at the same time allowing heat generated by viscous friction to escape through the solid [8].

Motion is produced by translating the lattice of tether sites at a fixed velocity. The tethers then drag the wall atoms, and the wall atoms drag the fluid with them. During the simulation we record the atomic positions, from which the contact angle may be estimated, and the force on the plate more precisely, the net force exerted by the fluid atoms on the plate atoms. In principle, the angle $\theta$ and the force $F$ are related by $F=2 W \gamma \cos \theta$, where $W$ is the plate width and $\gamma=2.8$ is the surface tension (and 2 comes from the two sides of the plate), but in practice the force measurement is the most reliable in that it is less susceptible to statistical fluctuations. We have also measured the local velocity and stress fields, but at the translation velocities considered here, thermal noise dominates and little information is present. One does however observe fluid slip at the contact line, as in $[6,8]$. To study the force or contact angle as a function of velocity, the system is first equilibrated, then a small positive plate velocity is applied and the system proceeds at this velocity until the force reaches a steady state. The velocity is then increased until a new steady state is reached. As the plate velocity increases, the fluid-fluid interface becomes increasingly distorted and eventually hits the top wall. This value is discarded, and the velocity is now decreased systematically until at large negative values the interface hits the bottom wall. This value is again discarded, and the velocity is increased back to zero. The force (or contact angle) vs. velocity plot is then examined.

\section{RESULTS}

First we consider an atomically smooth plate, where no hysteresis is anticipated. Indeed, the $f-v$ plot Fig. 2 is, within statistical fluctuations, a simple curve, with a single value of force depending only on the current velocity and not on the system's history. The change in the contact angle itself at least qualitatively follows the same trend: Fig. 3 gives snapshots of the three-phase region for various velocities. It is non-trivial to assign a specific value to the contact angle because of the fluctuations in the interface position itself at a given speed, as well as the the change in shape of the interface with distance from the contact line. Previous work [10] has addressed the latter issue, at least, and further work on analysis of the angle vs. velocity relationship and the interfacial shape is in progress. In this simulation, the Reynolds number based on the plate velocity and half-width of the cell is at maximum speed 1.2, while the capillary number extends up to 2.1.

Next we have considered two types of rough plate, obtained by displacing groups of solid atoms outward from their ideal-lattice positions. We either displace a periodic array of square regions as in [4] or, more realistically, groups of atoms chosen at random are displaced. In either case we find an open hysteresis loop; the result for the random case is shown in Fig.4, while the periodic case falls somewhat between the latter and the smooth case but is clearly multivalued. The qualitative features of the contact line shape are somewhat similar to the smooth-plate case, except that it appears more diffuse in a projected view because of a stronger variation along the direction of the contact line, as the latter attempts to follow the heterogeneity. Once again, further analysis is in progress.

\section{CONCLUSIONS}

Although these simulations have for the first time directly demonstrated the role of surface heterogeneity in producing hysteresis in a dynamic wetting process, they have only begun to explore the problem. We conclude by listing several further significant issues which are now under study, or which we will consider presently. 
1. We have considered only the steady wetting characteristics - the force or angle observed after a long displacement at a particular velocity. The pre-asymptotic behavior, i.e., the dynamic response to a change in wetting speed, is equally relevant. This topic amounts to exploring the interior of the hysteresis loop. Wetting behavior under cyclic operating conditions and its relation to dissipation have been considered theoretically [11], but only with simplified assumptions for the velocity dependence of the angle. Furthermore, the degree to which the phenomena studied here parallel the details of hysteresis in magnetic systems [12] is unclear.

2. We have simulated structural roughness alone, and only the simplest liquids. Various segregation phenomena may occur if the liquids are mixtures or if the molecules have specific end groups, or more generally if the chemistry of the system is non-trivial. The wetting of polymeric liquids is expected to be unusually interesting [13], even beyond the previously studied case of terraced wetting [9].

3. Analytic calculations for the motion of an interface near a single defect have shown a precursor of hysteretic behavior - a difference between the velocity seen at fixed force and the force measured at fixed velocity [5]. The calculations have been limited to the small-slope and lubrication approximations, however, and do not address multiple defects or systematic wettability variation at all. Since we can modify the solid surface at will, it is possible to explore the transition between isolated defects and various types of systematic heterogeneity.

\section{REFERENCES}

1. J. F. Padday, ed., Wetting, Spreading and Adhesion (Academic, 1978).

2. E. B. Dussan V., Annu. Rev. Fluid Mech. 11, 371 (1979); P. G. de Gennes, Rev. Mod. Phys. 57, 827 (1985); L. Leger and J. F. Joanny, Rep. Prog. Phys. 55, 431 (1992).

3. R. E. Johnson Jr. and R. H. Dettre, in Surface and Colloid Science, ed. E. Matejevic, 2, 85 (1967).

4. L. W. Schwartz and S. Garoff, Langmuir 1, 219 (1985).

5. J. F. Joanny and P. G. de Gennes, J. Chem. Phys. 81, 552 (1984); E. Raphael and P. G. de Gennes, J. Chem. Phys. 90, 7577 (1989); J. F. Joanny and M. O. Robbins, J. Chem. Phys. 92, 3206 (1990); A. Paterson, M. Fermigier, P. Jennfer and L. Limat, Phys. Rev. E 51, 1291 (1995).

6. J. Koplik, J. R. Banavar and J. F. Willemsen, Phys. Fluids A 1, 781 (1989).

7. J. Koplik and J. R. Banavar, Annu. Rev. Fluid Mech. 27, 257 (1995).

8. P. A. Thompson and M. O. Robbins, Phys. Rev. Lett. 63, 766 (1989).

9. J.-x. Yang, J. Koplik and J. R. Banavar, Phys. Rev. A 46, 7738 (1992); J. A. Nieminen and T. Ala-Nissila, Phys. Rev. E 49, 4228 (1994); U. d'Ortona, J. De Coninck, J. Koplik and J. R. Banavar, Phys. Rev. E 53, 562 (1996).

10. R. G. Cox, J. Fluid Mech. 168, 169 (1986); J. A. Marsh, S. Garoff and E. B. Dussan V., Phys. Rev. Lett. 70, 2778 (1993).

11. G. W. Young and S. H. Davis, J. Fluid Mech. 174, 327 (1987).

12. K. Dahmen, et al., J. Appl. Phys. 75, 5946 (1994), and references therein.

13. K. R. Willson and S. Garoff, Colloids and Surfaces 89, 263 (1994). 


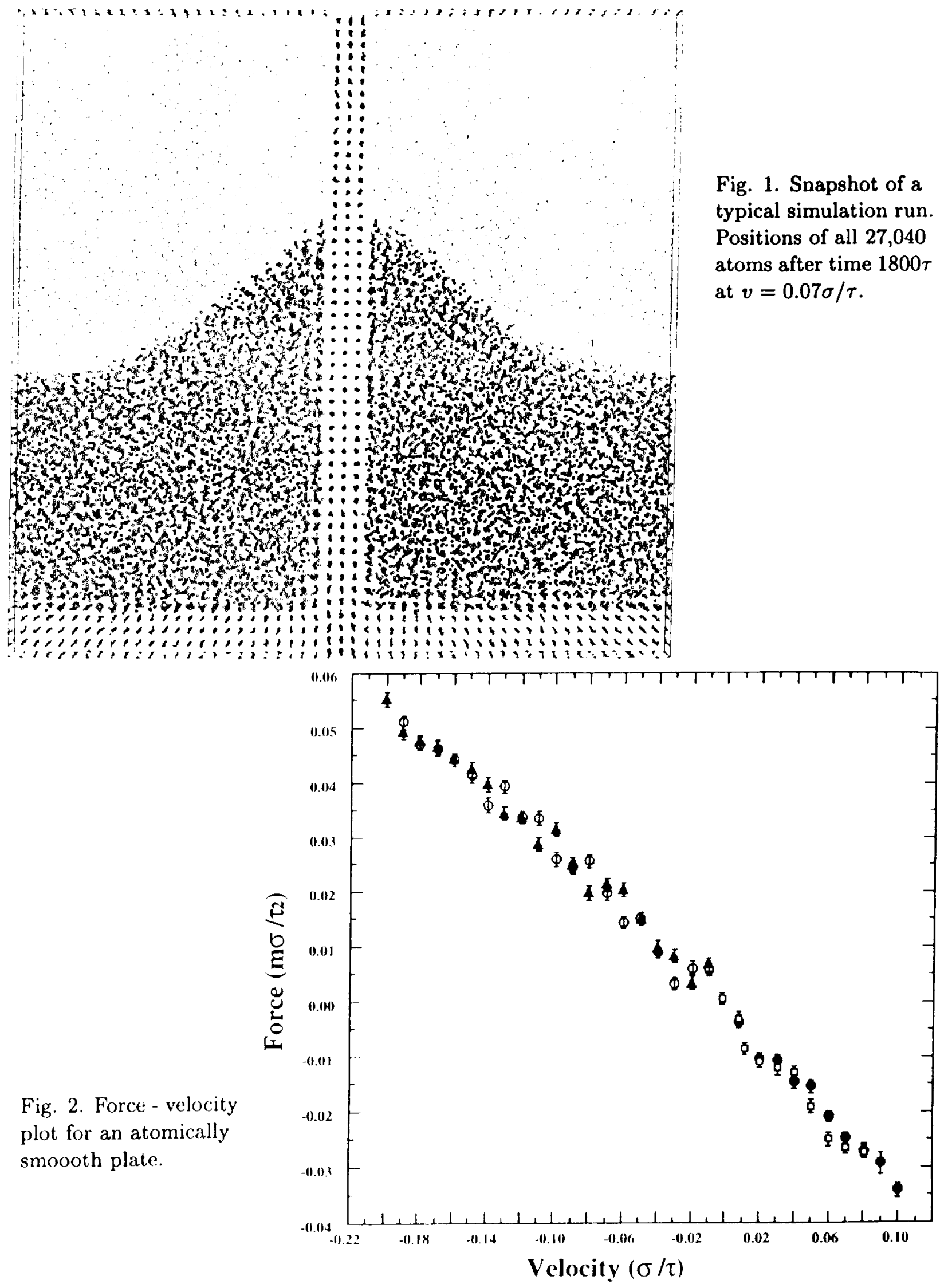



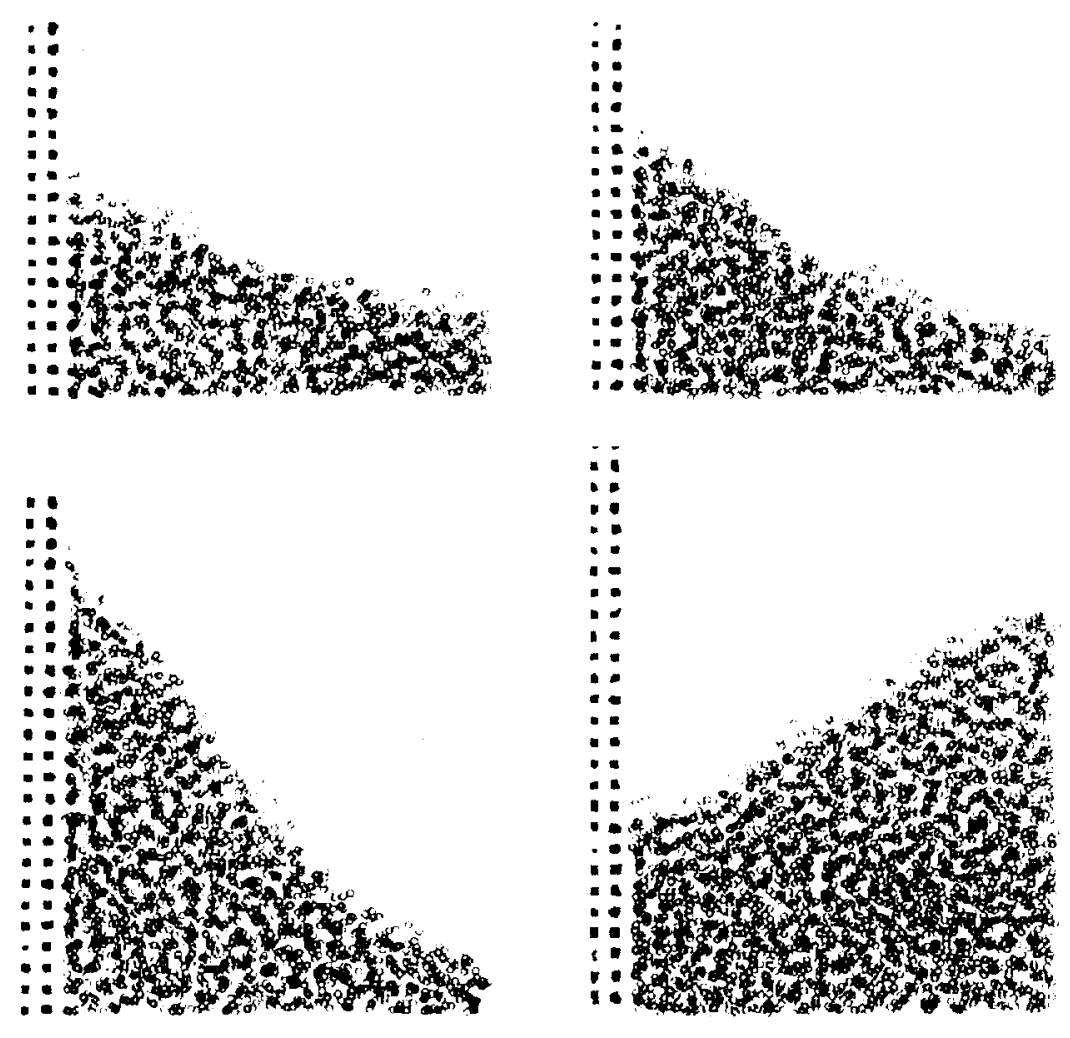

Fig. 3. Variation of instantaneous interface shape with velocity: $v=0.03$ (a), 0.06 (b), 0.09 (c), $-0.20 \sigma / \tau$ (d).

Fig. 4. Force - velocity plot for a randomly rough plate.

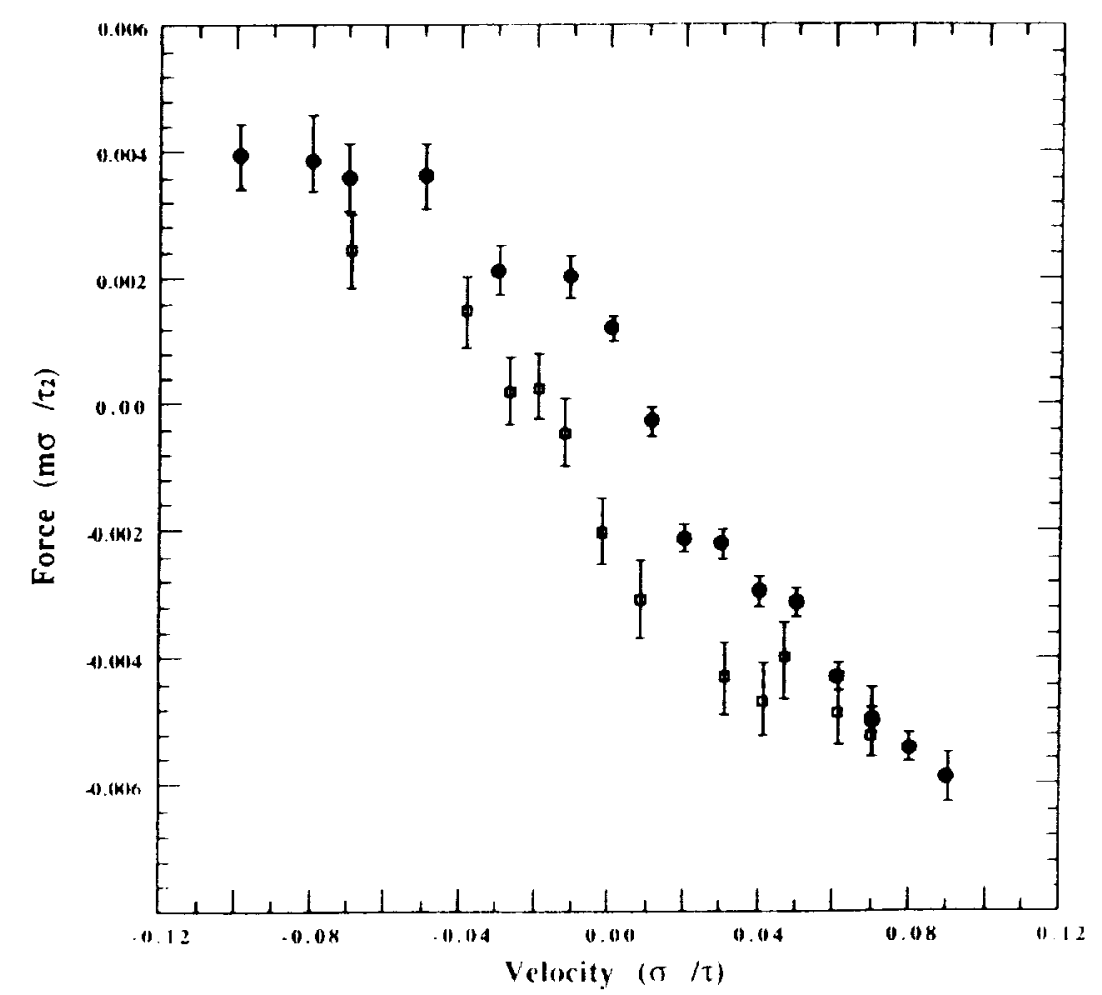

